\title{
Adaptive Steganography using 3D Color Texture Feature
}

\author{
P.Pavan Kumar, L.Suneel, Nagaraja Kumar Pateti, A.M.Srinivasacharyulu
}

\begin{abstract}
Human vision framework is commonly an emotional recognition which differs according to people. Intricacy of a picture assumes huge job while verifying information in to it. In this paper another steganography approach is introduced which uses joined 3D Color Texture Feature (CTF) to distinguish complex districts of picture for information stowing away so visual assault to identify shrouded message turns out to be very testing. Recurrence area is utilized to shroud the information in these chose complex areas by means of Discrete Cosine Transform (DCT). These sorts of zones are initially boisterous and separating additional data is difficult. Each picture has diverse multifaceted nature levels and spatial districts, and since information covering up is legitimately reliant on it, so the steganography framework ends up versatile. The outcome demonstrates that proposed versatile strategy gives secure message stowing away while keeping up subtlety quality and high implanting limit. Last spread pictures keeps up PSNR estimation of over 50. Inserting limit is around multiple times higher in contrast with comparative calculation which uses Gray Level Co-event Matrices (GLCM) highlight to recognize complex districts of pictures for information covering up.
\end{abstract}

Keywords-versatile steganography, unpredictability examination, shading surface element, CTF, GLCM surface, DCT

\section{INTRODUCTION}

These days advanced media is the greatest wellspring of information correspondence. Security of data ends up urgent when the information being moved is private [2]. As a result of the wide web access there are tremendous dangers, for example, danger of information spillage, debasement and devastation which thusly upgrades a gigantic measure of hazard on private information [1]. So as to take care of this issue Steganography is very useful.

In Steganography data can be covered up in bearers, for example, content records, pictures, sound and video [10]. Spatial and recurrence are fundamentally two kinds of areas in which information stowing away is practiced. In spatial area most prevalent systems are least noteworthy piece (LSB) and most huge piece (MSB) though in recurrence space first spread picture is changed into recurrence space utilizing changes like DCT, DWT and SVD and so on and afterward

Revised Version Manuscript Received on September 10, 2019.

P.Pavan Kumar, Associate Professor, Department of Electronics and Communication Engineering, CMR Institute of Technology, Hyderabad, Telangana, India.(E.Mail:pavanu.857@gmail.com)

L.Suneel, Assistant Professor, Department of Electronics and Communication Engineering, CMR Institute of Technology, Hyderabad, Telangana, India.(E-Mail: suneel.430@gmail.com)

Nagaraja Kumar Pateti, Assistant Professor, Department of Electronics and Communication Engineering, CMR Institute of Technology, Hyderabad, Telangana, India.(E-Mail: nagaraja1609@gmail.com)

A.M.Srinivasacharyulu, Assistant Professor, Department of Electronics and Communication Engineering, CMR Institute of Technology, Hyderabad, Telangana, India.(E-Mail: aacharyasrinivas@gmail.com) information stowing away is performed in like manner [4]. Picture unpredictability examination gives crucial viewpoints about it in spatial space. Visual assaults can be countered all around effectively if the data is covered up in such locales where any little changes in pixels are exceptionally difficult to distinguish. As per the picture highlights, thinking about the presence of dark levels, pixel dissemination and dim level pixel number; picture unpredictability is communicated by the dim spatial appropriation, appearance of dim level, alongside the presence of the objective [15]. Following this presentation a concise foundation is given to calculation to separate 3D shading surface component (CTF) from spread picture to dissect intricacy in segment 2 . The useful square outline and stream graph of the calculation alongside top to bottom applied rationale is portrayed in area 3. Aftereffects of the proposed strategy utilizing CTF and examinations with surface component are given in segment 4 , while segment 5 at last finishes up the total work and recommends extra territories of ad lib.

\section{RELATED WORK}

A relative investigation of various novel takes a shot at steganography was done to comprehend current patterns in steganography. As it is vital to actualize right methodology for protected and secure information covering up. G. Sreenath [7] has proposed a surface based secure information transmission approach. Information is shrouded utilizing surface combined reversible steganography strategy and equality checking which depends on tree calculation. Proposed calculation in paper is professed to give more elevated amount of security in all methods.

C. Zheng, M. Shen, X. M. Li and X. Zhang [8] exhibited another steganography strategy dependent on profound learning of convolutional neural system $(\mathrm{CNN})$. Spread picture is partitioned in to numerous sub pictures to get three unique kinds of pictures by change of focus pixel. Pixel adjustment cost is assessed according to installing doubt. CNN model is prepared to assess implanting doubt. According to examinations introduced in the paper proposed calculation is aggressive and CNN can be connected in steganography.

D. R. Herrera-Moro, R. Rodriguez-Colin and C. Feregrino-Uribe [9] proposed a versatile steganography technique which depends on surface highlights. According to proposed steganography calculation which is named as setting, chooses the pixels from the surface districts which are non-homogeneous to conceal the information.

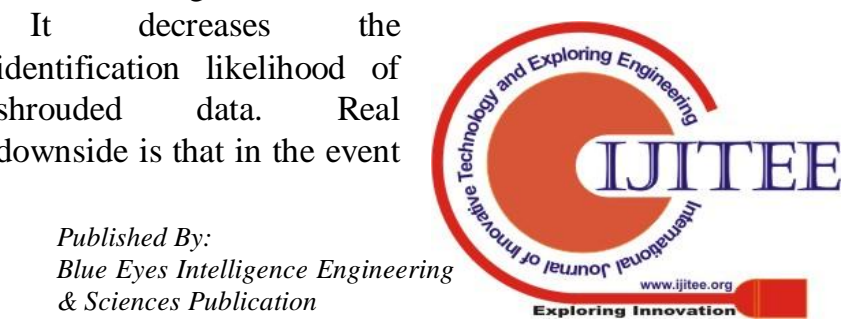


that any channel is connected, at that point message is lost since least huge piece (LSB) is utilized to store the picture.

\section{PROPOSED METHODOLOGY}

Various methodologies are proposed by creators for discrete extraction of surface data to conceal the information in such areas where likelihood of recognition by means of visual assault is low. Almost certainly, surface data gives indispensable data about the intricacy of areas yet it totally relies on the dim spatial circulation as it were. As most of pictures which we experience in everyday life contains shading data too, so on the off chance that this shading data can likewise be coordinated while assessing surface data, at that point it can improve the multifaceted nature investigation which thus fundamentally upgrades the security and inserting limit while keeping up the visual nature of spread picture.

In this paper another calculation is proposed to separate two kinds of data in a coordinated way which are shading and surface as a solitary substance. Fundamentally, shading picture contains 3 planes which are Red, Green and Blue abridged as RGB and message can be implanted in every one of the planes adaptively according to the intricacy examination of the individual locales.

A practical square chart of the proposed framework is appeared in the Fig 1.

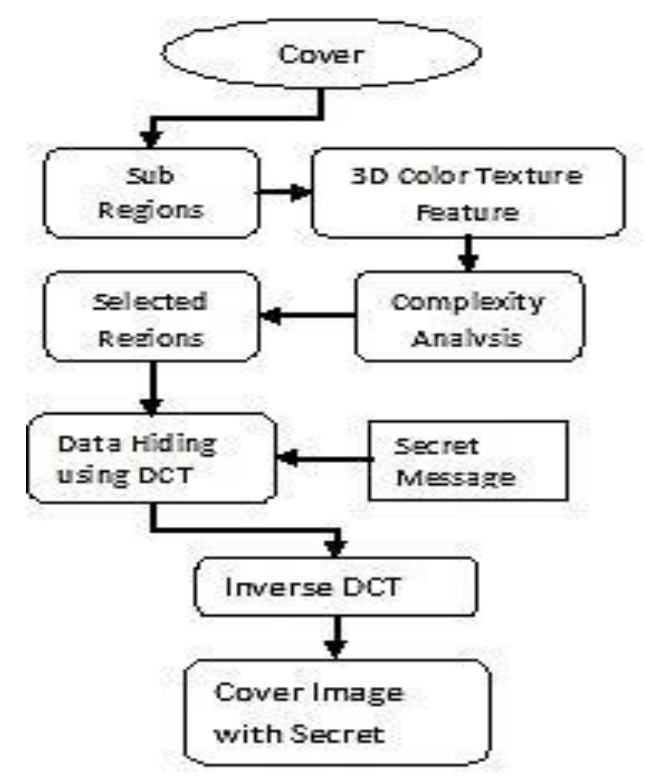

Figure 1: Functional square chart of proposed framework.

\section{Picture Sub-Regions}

As a matter of first importance venture before unpredictability investigation is to isolate the picture in to consistently little individual ( $\mathrm{m} \mathrm{x} \mathrm{n}$ ) squares which parts the entire picture into sub districts since it is computationally simple to break down little areas. It improves the installing limit and join versatile nature also. In this paper pictures which are tried against the proposed calculation are of the measurement $512 \times 512 \times 3$ (lines $x$ sections $x$ planes). So the picture is separated into $8 \times 8$ squares which thusly gives 4096 sub locales to every one of the three planes. It isn't mandatory to isolate the picture into squares of $8 \times 8$ measurement as it were. As a matter of fact it very well may be partitioned in to

sub-squares of any measurements and it thoroughly fluctuates upon application. There is a tradeoff between security, limit and calculation intricacy while choosing sub square measurements. Littler square outcomes in higher inserting limit however alongside surprising expense of computational multifaceted nature and the other way around. Littler squares additionally prompts expanded likelihood of recognition for shrouded message and lower the PSNR esteems. The $8 \times 8$ worth is experimental establishing to test the calculation while keeping up the equalization among tradeoffs. Presently these sub districts can be independently broke down and to shroud mystery information in to chosen areas according to edge conditions.

\section{Complexity Analysis Using 3D CTF}

Co-event networks of 14 measurable highlights for surface grouping was characterized by Harlick [22]. 3 out 14 are utilized in view of solid co-connection among them regarding factual highlights. CLCM (shading level co-event lattice) grid depends on GLCM and conditions characterizing it are given beneath from condition (1) to (3). For coarse surfaces estimation of differentiation from condition (1) is enormous and generally it's little. From condition (2) connection worth is bigger for comparable pictures while its worth is littler for bigger multifaceted nature. For coarse surface Energy esteems is littler and it's bigger generally from condition (3) [15].

$$
\begin{aligned}
& \text { Contrast }=\sum_{i, j}|i-j|^{2} p(i, j) \\
& \text { Correlation }=\sum_{i, j} \frac{(i-\mu i)(j-\mu j) p(i, j)}{\sigma_{i} \sigma_{j}} \\
& \text { Energy }=\sum_{i, j} p(i, j)^{2}
\end{aligned}
$$

How often a dark pixel is connected to neighboring pixel is determined by these arrangement of conditions. Separation $d$ (between pixel of intrigue and adjoining pixel) and precise spatial relationship theta $(\Theta=0,90,45$ and 135) for a picture can shifted as needs be. In the proposed calculation $d=1$ and $\Theta=0$ is utilized for calculation.

TABLE I. 6x3 CTF matrix for 8x8 block

\begin{tabular}{|c|c|c|c|c|}
\hline \multirow{2}{*}{ Sr. } & \multirow{2}{*}{ Planes } & \multicolumn{3}{|c|}{ Features } \\
\cline { 3 - 5 } & & Contrast & Correlation & Energy \\
\hline 1 & Red-Green & 0.5833 & 0.0000 & 0.5139 \\
\hline 2 & Red-Blue & 0.5833 & 0.0000 & 0.5139 \\
\hline 3 & Green-Red & 0.7500 & 0.0000 & 0.6250 \\
\hline 4 & Green-Blue & 0.0000 & 0.0000 & 1.0000 \\
\hline 5 & Blue-Red & 0.7500 & 0.0000 & 0.6250 \\
\hline 6 & Blue-Green & 0.0000 & 0.0000 & 1.0000 \\
\hline
\end{tabular}

While ascertaining GLCM highlights segments from every one of the three planes $(\mathrm{R}, \mathrm{G}, \mathrm{B})$ are utilized that is the reason resultant lattice is known as shading level co-event grid (CLCM) and the data removed contains the surface just as shading data from each of the three planes; subsequently known as 3D shading surface future (CTF). 


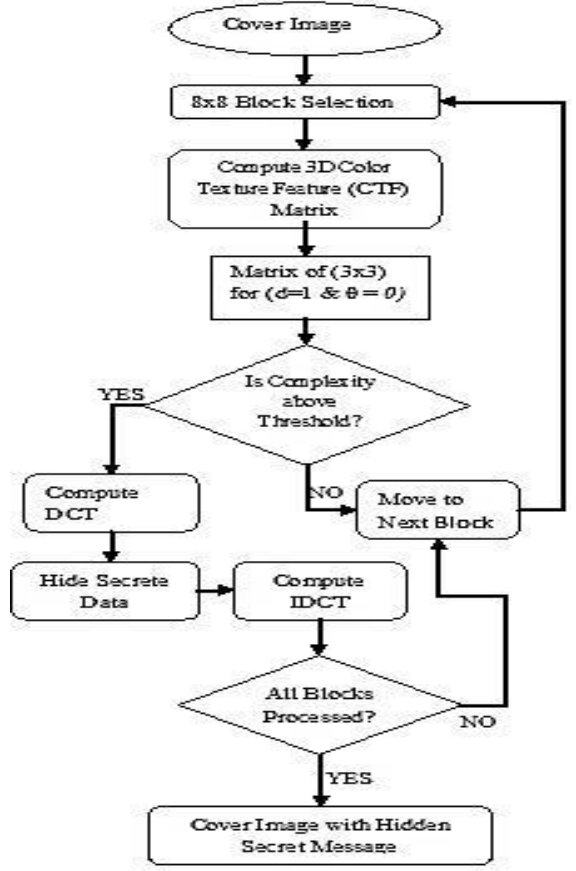

Figure 2: Stream diagram of proposed framework.

An example of $6 \times 3$ CTF frameworks for a $8 \times 8$ window of arbitrary square from a picture is appeared in Table I. In the table each of the 3 GLCM highlights specifically differentiation, connection and vitality registered separately according to condition 1 to 3 utilizing variables $\Theta=0$ and $d=$ 1. For red plane estimations of red-green and red-blue are found the middle value of. Thus estimations of different planes are determined and the $6 \times 3$ lattice is diminished to $3 \times 3$ network. In the proposed calculation limit esteems for each of the three properties are considered precisely at center, in spite of the fact that it can likewise be shifted by application. The squares which are having intricacy previously mentioned limit are chosen for information stowing away. Various squares can be qualified for information escaping every one of the three planes (R, G and B) as the intricacy esteems can vary and absolutely reliant on the square's component which is under investigation.

A stream graph for proposed calculation is likewise appeared in Fig. 2. To register the CTF framework of complete picture all pixels are chosen individually with an area of $8 \times 8$ successively from each of the three planes. Unpredictability qualification to conceal information is chosen according to CTF lattice esteems, in the event that they are above edge, at that point just the square is chosen for mystery information covering up.

\section{A. DCT, Data Hiding and Data Extraction}

DCT is an appropriate recurrence area to shroud the information as it is lossless and invertible [21]. Rather than changing entire picture in to recurrence area, just the chose squares which are qualified for information stowing away are changed. To insert the mystery message which is in double configuration; $\mathrm{x}, \mathrm{y}(4,4)$ area is chosen from the square of $8 \times 8$. As this area is in mid band and an accurate harmony among subtlety and strength of a picture. To conceal the parallel message the pixel at the referenced area is changed over to a negative worth if mystery message worth is 1 and made positive generally. Just one worth is covered up in each chose squares. In the wake of concealing mystery message the square is reconverted to its unique from utilizing IDCT.

To extricate the information from this picture a similar strategy is pursued according to stream graph referenced in Fig. 2. Just at the spot of information concealing the extremity of the pixel at the area 4, 4 is checked and mystery message is remade. No index entries found.

\section{RESULTS AND DISCUSSION}

Results are explored on standard pictures, for example, Lena, Mandrill and Peppers and so forth. Fig. 3 and 4 demonstrates the first and stego picture of Mandrill and Airplane individually. Information is covered up in every single qualified square to completely test the subtlety against the inserting limit.
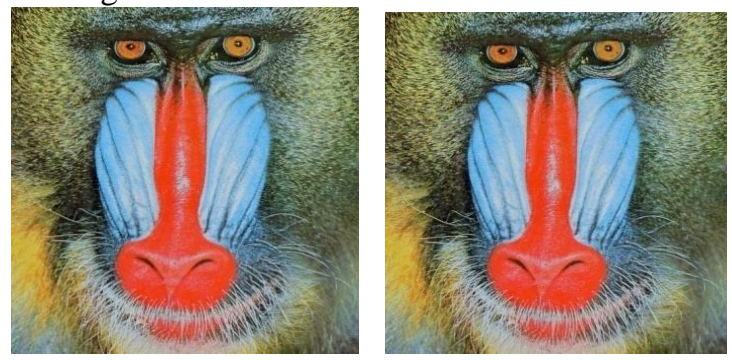

Figure 3:Mandrill, unique picture (left) and Stego picture (right)

Installing limit, mystery message extraction rate and impalpability of picture (PSNR) is appeared in Fig. 5, 6 and 7 separately. An examination is appeared against the comparable calculation which figures multifaceted nature of the picture just dependent on surface component utilizing GLCM. Though during examination of the installing information, for equivalent grounds of assessment limit of GLCM strategy is increased by a factor of 3 in light of the fact that GLCM is connected on grayscale picture just while in $3 \mathrm{D} C \mathrm{CTF}$ it is registered on 3 planes ( $\mathrm{R}, \mathrm{G}$ and $\mathrm{B})$. It very well may be obviously broke down that proposed technique furnish twice inserting limit with PSNR estimation of over 50 alongside better extraction level of mystery information from stego picture.
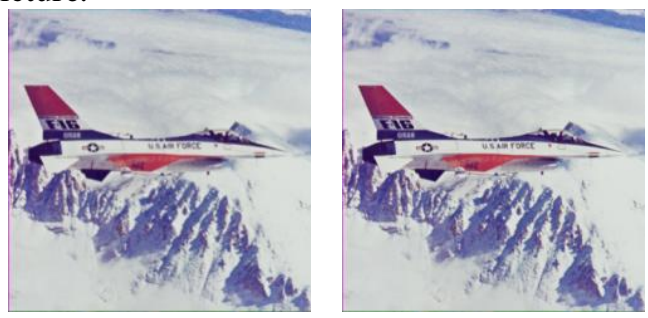

Figure 4: Airplane, original image (left) and Stego image (right)

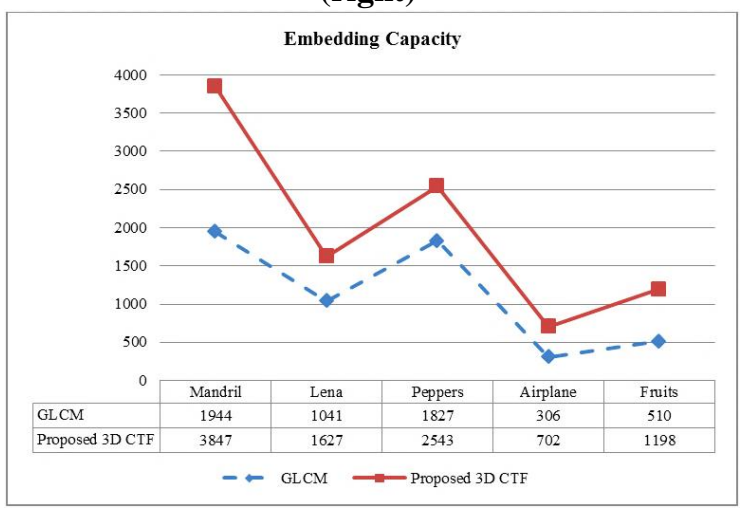

Figure 5: Embedding capacity of GLCM v/s 3D CTF

Blue Eyes Intelligence Engineering 


\section{CONCLUSION}

Another steganography technique is proposed in this paper to shroud the mystery information by means of versatile determination of squares through multifaceted nature investigation of the picture utilizing 3D CTF.

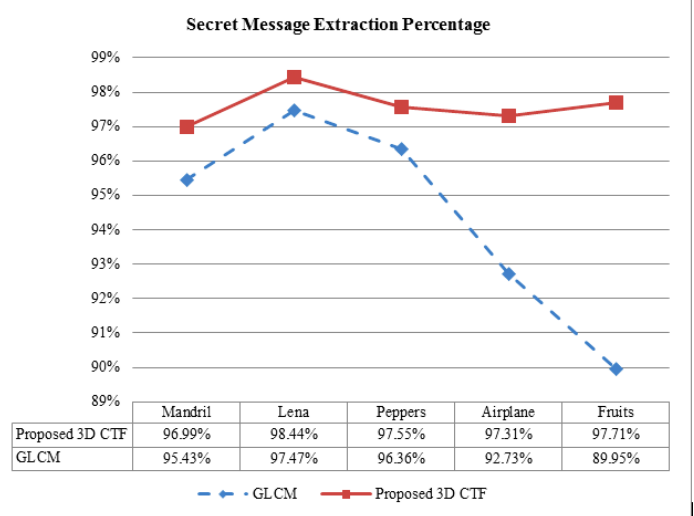

Figure 6: Secret message extraction (\%) of GLCM v/s 3D CTF

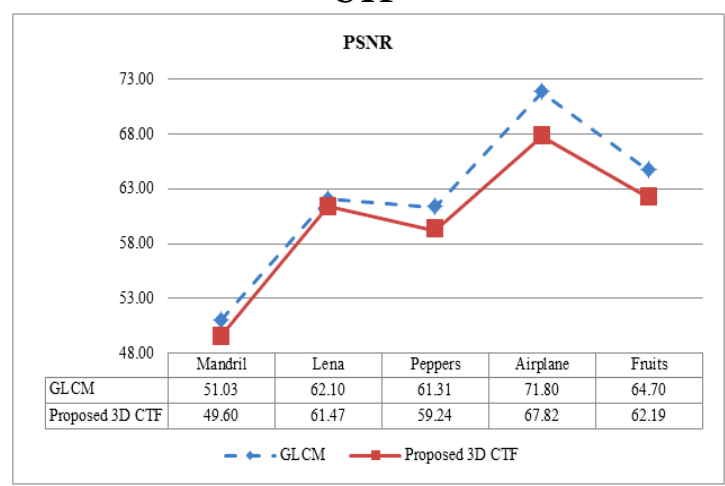

Figure 7: Stego picture PSNR of GLCM v/s 3D CTF

Calculation partitions the picture into square of $8 \times 8$ and intricacy is assessed autonomously for every one of the 3 planes. Information is shrouded uniquely in chosen squares who clears the qualification edge. DCT is utilized to change just chosen squares in to recurrence area to conceal the mystery message. Proposed calculation gives just about multiple times the implanting limit with improved mystery message extraction in contrast with GLCM technique while keeping up the impalpability quality over 50 as far as PSNR.

Proposed plan gives new progression in the field of steganography by giving a versatile square choice strategy dependent on spatial intricacy by means of 3D CTF. In future, proposed strategy can be assessed against different shading models and edge enhancement to improve framework execution. Another zone of impromptu creation can be bringing down the computational multifaceted nature to accelerate the calculation

\section{REFERENCES}

1. Ridhima Grover, D K Chauhan, Dinesh KumarYadav, Suraj Kamya, "Adaptive Steganography via Image Complexity Analysis using 3D Color Texture Feature" 3rd IEEE International Conference on Innovative Applications of Computational Intelligence on Power, Energy and Controls with their on Humanity (CIPECH 2018), KIET Ghaziabad.

2. M. Ulker and B. Arslan, "A novel secure model: Image steganography with logistic map and secret key," 2018 6th International Symposium on Digital Forensic and Security (ISDFS), Antalya, 2018, pp. 1-5.

3. D. R. I. M. Setiadi, H. A. Santoso, E. H. Rachmawanto and C. A. Sari, "An improved message capacity and security using divide and modulus function in spatial domain steganography," 2018 International Conference on Information and Communications Technology (ICOIACT), Yogyakarta, 2018, pp. 186-190.
4. Y. P. Astuti, D. R. I. M. Setiadi, E. H. Rachmawanto and C. A. Sari, "Simple and secure image steganography using LSB and triple XOR operation on MSB," 2018 International Conference on Information and Communications Technology (ICOIACT), Yogyakarta, 2018, pp. 191-195.

5. P. Johri, A. Mishra, S. Das and A. Kumar, "Survey on steganography methods (text, image, audio, video, protocol and network steganography)," 2016 3rd International Conference on Computing for Sustainable Global Development (INDIACom), New Delhi, 2016, pp. 2906-2909.

6. G. L. Smitha and E. Baburaj, "A survey on image steganography based on block-based edge adaptive based on Least Significant Bit Matched Revisited (LSBMR) algorithm," 2016 International Conference on Control, Instrumentation, Communication and Computational Technologies (ICCICCT), Kumaracoil, 2016, pp. 132-139.

7. G. Sreenath et al., "Texture based secure data transmission," 2017 International conference of Electronics, Communication and Aerospace Technology (ICECA), Coimbatore, 2017, pp. 38-42.

8. C. Zheng, M. Shen, X. M. Li and X. Zhang, "Texture adaptive steganography via Convolutional Neural Networks," 2017 3rd IEEE International Conference on Computer and Communications (ICCC), Chengdu, 2017, pp. 1416-1420.

9. D. R. Herrera-Moro, R. Rodriguez-Colin and C. Feregrino-Uribe, "Adaptive Steganography based on textures," 17th International Conference on Electronics, Communications and Computers (CONIELECOMP'07), Cholula, Puebla, 2007, pp. 34-34.

10. P. Joseph and S. Vishnukumar, "A study on steganographic techniques," 2015 Global Conference on Communication Technologies (GCCT), Thuckalay, 2015, pp. 206-210.

11. J. Bhadra, M. K. Banga and M. V. Murthy, "Securing data using elliptic curve cryptography and least significant bit steganography," 2017 International Conference On Smart Technologies For Smart Nation (SmartTechCon), Bangalore, 2017, pp. 1460-1466.

12. M. Douglas K. Bailey M. Leeney K. Curran "An overview of steganography techniques applied to the protection of biometric data" Multimedia Tools and Applications pp. 1-41 2017.

13. M. Saidi, H. Hermassi, R. Rhouma and S. Belghith, "LSB-hamming based chaotic steganography (LH-Steg)," 2017 12th International Conference for Internet Technology and Secured Transactions (ICITST), Cambridge, 2017, pp. 29-34.

14. P. Khanzadi, B. Majidi \& E. Akhtarkavan , “A Novel Metric for Digital Image Quality Assessment using Entropy-Based Image Complexit”, IEEE 4th International Conference on Knowledge-Based Engineering and Innovation (KBEI), Dec. 22QG, 2017.

15. M. Liu, K. Gu, G. Zhai and P. Le Callet, "Visual saliency detection via image complexity feature," 2016 IEEE International Conference on Image Processing (ICIP), Phoenix, AZ, 2016, pp. 2777-2781. S. Yang, et al., "Objective Image Quality Assessment based on Image Complexity and Color Similarity", 2013 Fourth World Congress on Software Engineering.

16. M. Chacon, et al., "A Fuzzy Approach on Image Complexity Measure", Computación y Sistemas Vol. 10 No. 3, 2007, pp 268-284.

17. H. Yu and S. Winkler, "Image Complexity And Spatial Information", 2013 Fifth International Workshop on Quality of Multimedia Experience (QoMEX)

18. M. Benco, R. Hudec, P. Kamencay, M. Zachariasova and S. Matuska, "An Advanced Approach to Extraction of Color Texture Features Based on GLCM", International Journal of Advanced Robotic Systems,pp.1-8, 2014

19. Mario I., Chacon M., Ama D. and Corral S., "Image Complexity Measure: a Human Criterion Free Approach", NAFIPS 2005 - 2005 Annual Meeting of the North American Fuzzy Information Processing Society.

20. Matthieu Perreira da Silva, Vincent Courboulay, Pascal Estraillier. “ Image Complexity Measure Based On Visual Attention". IEEE International Conference on Image Processing -ICIP, Sep 2011, Bruxelles, Belgium. pp.3281-3284, 2011

21. X. Song, S. Wang and X. Niu, "An Integer DCT and Affine Transformation Based Image Steganography Method," 2012 Eighth International Conference on Intelligent Information Hiding and Multimedia Signal Processing, Piraeus, 2012, pp. 102-105.

22. A. Girdhar and V. Kumar, "Comprehensive survey of 3D image steganography techniques," in IET Image Processing, vol. 12, no. 1, pp. $1-10,12018$.

23. Haralick R M, Shanmugam K, Dinstein Its 'Hak Textural features for image classification, Systems, Man and Cybernetics, IEEE Transactions, vol. SMC-3, no. 6, pp. 610-621, 1973. 